\title{
Errata for Computing the Top Betti Numbers of Semialgebraic Sets Defined by Quadratic Inequalities in Polynomial Time (DOI: 10.1007/s10208-005-0208-8)
}

Saugata Basu

Corollary 1.3 has been amended. The text is reproduced here. The original Section 4.3 has been deleted. Substantial changes have been made to Sections 6 and 7, and the text is reproduced here on the following pages.

Corollary 1.3. Let $\mathrm{R}$ be a real closed field and let $S \subset \mathrm{R}^{k}$ be defined by

$$
\bigwedge_{P \in \mathcal{P}_{1}} P=0 \bigwedge_{P \in \mathcal{P}_{2}} P>0 \bigwedge_{P \in \mathcal{P}_{3}} P<0
$$

with $\operatorname{deg}(P) \leq 2$ for each $P \in \bigcup_{i=0,1,2} \mathcal{P}_{i}$, and $\# \bigcup_{i=0,1,2} \mathcal{P}_{i}=s$.

Then, for all $\ell \geq 0$,

$$
b_{k-\ell}(S) \leq\left(\begin{array}{l}
s \\
\ell
\end{array}\right) k^{O(\ell)} \text {. }
$$

In the following proof, as well as later in the paper, we will extend the ground field $\mathrm{R}$ by infinitesimal elements. We denote by $\mathrm{R}\langle\zeta\rangle$ the real closed field of algebraic Puiseux series in $\zeta$ with coefficients in $\mathrm{R}$ (see [9] for more details). The sign of a Puiseux series in $\mathrm{R}\langle\zeta\rangle$ agrees with the sign of the coefficient of the lowest degree term in $\zeta$. This induces a unique order on $\mathrm{R}\langle\zeta\rangle$ which makes $\zeta$ infinitesimal: $\zeta$ is positive and smaller than any positive element of R. When $a \in \mathrm{R}\langle\zeta\rangle$ is bounded from above and below by some elements of $\mathrm{R}, \lim _{\zeta}(a)$ is the constant term of $a$, obtained by substituting 0 for $\zeta$ in $a$. Given a semialgebraic set $S$ in $\mathrm{R}^{k}$, the extension of $S$ to $\mathrm{R}^{\prime}$, denoted $\operatorname{Ext}\left(S, \mathrm{R}^{\prime}\right)$, is the semialgebraic subset of $\mathrm{R}^{\prime k}$ defined by the same quantifier-free formula that defines $S$. The $\operatorname{set} \operatorname{Ext}\left(S, \mathrm{R}^{\prime}\right)$ is

Online publication: April 23, 2008. 
well defined (i.e., it only depends on the set $S$ and not on the quantifier-free formula chosen to describe it). This is an easy consequence of the transfer principle (see, for instance, [9]).

Proof of Corollary 1.3. Let $0<\delta \ll \varepsilon \ll 1$ be infinitesimals. We first replace the set $S$ by the set $S^{\prime} \subset \mathrm{R}\langle\varepsilon\rangle^{k}$ defined by $S^{\prime}=\operatorname{Ext}(S, \mathrm{R}\langle\varepsilon\rangle) \cap \bar{B}_{k}(0,1 / \varepsilon)$, where $\bar{B}_{k}(0, r)$ denotes the closed ball of radius $r$ centered at the origin. It follows from Hardt's triviality theorem for semialgebraic mappings [19] that $b_{i}(S)=b_{i}\left(S^{\prime}\right)$ for all $i \geq 0$. We then replace $S^{\prime}$ by the set $S^{\prime \prime} \subset \mathrm{R}\langle\varepsilon, \delta\rangle^{k}$ defined by

$$
\begin{aligned}
\bigwedge_{P \in \mathcal{P}_{1}} P & \leq 0 \wedge-P \leq 0 \bigwedge_{P \in \mathcal{P}_{2}}-P+\delta \leq 0 \bigwedge_{P \in \mathcal{P}_{3}}-P-\delta \\
& \leq 0 \bigwedge \varepsilon^{2}\left(X_{1}^{2}+\cdots+X_{k}^{2}\right)-1 \leq 0 .
\end{aligned}
$$

It follows from Hardt's triviality again that $b_{i}\left(S^{\prime}\right)=b_{i}\left(S^{\prime \prime}\right)$ for all $i \geq 0$. Now apply Theorem 1.1. 


\section{Computing the Cohomology Groups of a Basic Semi-Algebraic Set Defined by Homogeneous Quadratic Inequalities}

In this section, we will show how to effectively compute the spectral sequence described in the previous section.

Let $\mathcal{P}=\left(P_{1}, \ldots, P_{s}\right) \subset \mathrm{R}\left[X_{0}, \ldots, X_{k}\right]$ be a $s$-tuple of quadratic forms. For any subset $\mathcal{Q} \subset \mathcal{P}$, we denote by $T_{\mathcal{Q}} \subset \mathbf{S}^{k}$, the semi-algebraic set,

$$
T_{\mathcal{Q}}=\bigcup_{P \in \mathcal{Q}}\left\{x \in \mathbf{S}^{k} \mid P(x) \leq 0\right\},
$$

and let

$$
S=\bigcap_{P \in \mathcal{P}}\left\{x \in \mathbf{S}^{k} \mid P(x) \leq 0\right\} .
$$

We denote by $C^{\bullet}\left(\mathcal{H}\left(T_{\mathcal{Q}}\right)\right)$ the co-chain complex of a cellular subdivision, $\mathcal{H}\left(T_{\mathcal{Q}}\right)$ of $T_{\mathcal{Q}}$, which is to be chosen sufficiently fine (to be specified later).

We first describe for each subset $\mathcal{Q} \subset \mathcal{P}$ with $\# \mathcal{Q}=\ell<k$, a complex, $\mathcal{M}_{\mathcal{Q}}$, and natural homomorphisms,

$$
\psi_{\mathcal{Q}}: C^{\bullet}\left(\mathcal{H}\left(T_{\mathcal{Q}}\right)\right) \rightarrow \mathcal{M}_{\mathcal{Q}}^{\bullet},
$$

which induce isomorphisms,

$$
\psi_{\mathcal{Q}}^{*}: H^{*}\left(C^{\bullet}\left(\mathcal{H}\left(T_{\mathcal{Q}}\right)\right)\right) \rightarrow H^{*}\left(\mathcal{M}_{\mathcal{Q}}^{\bullet}\right) .
$$

Moreover, for $\mathcal{B} \subset \mathcal{A} \subset \mathcal{P}$ with $\# \mathcal{A}=\# \mathcal{B}+1<k$, we construct a homomorphism of complexes,

$$
\varphi_{\mathcal{A}, \mathcal{B}}: \mathcal{M}_{\mathcal{A}}^{\bullet} \rightarrow \mathcal{M}_{\mathcal{B}},
$$

such that the following diagram commutes,

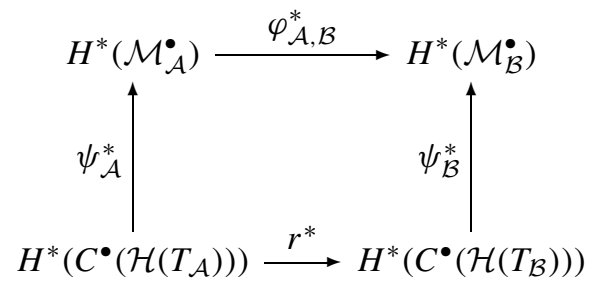

where $\varphi_{\mathcal{A}, \mathcal{B}}^{*}$ and $r^{*}$ are the induced homomorphisms of $\varphi_{\mathcal{A}, \mathcal{B}}$ and the restriction homomorphism $r$ respectively.

Now, consider a fixed subset $\mathcal{Q} \subset \mathcal{P}$, which without loss of generality we take to be $\left\{P_{1}, \ldots, P_{\ell}\right\}$. Let

$$
P=\left(P_{1}, \ldots, P_{\ell}\right): \mathrm{R}^{k+1} \rightarrow \mathrm{R}^{\ell}
$$

denote the corresponding quadratic map. 
As in the previous section, let $\mathrm{R}^{\mathcal{Q}}=\mathrm{R}^{\ell}$, and

$$
\Omega_{\mathcal{Q}}=\left\{\omega \in \mathrm{R}^{\ell}|| \omega \mid=1, \omega_{i} \leq 0,1 \leq i \leq \ell\right\} .
$$

Let $B_{\mathcal{Q}} \subset \Omega_{\mathcal{Q}} \times \mathbf{S}^{k}$ be the set defined by,

$$
B_{\mathcal{Q}}=\left\{(\omega, x) \mid \omega \in \Omega_{\mathcal{Q}}, x \in \mathbf{S}^{k} \text { and } \omega P(x) \geq 0\right\},
$$

and we denote by $\varphi_{1, \mathcal{Q}}: B_{\mathcal{Q}} \rightarrow \Omega_{\mathcal{Q}}$ and $\varphi_{2, \mathcal{Q}}: B_{\mathcal{Q}} \rightarrow \mathbf{S}^{k}$ the two projection maps.

For each subset $\mathcal{Q}^{\prime} \subset \mathcal{Q}$ we have a natural inclusion $\Omega_{\mathcal{Q}^{\prime}} \hookrightarrow \Omega_{\mathcal{Q}}$. Let

$$
h_{\mathcal{Q}}: \Delta_{\mathcal{Q}} \rightarrow \Omega_{\mathcal{Q}}
$$

be a semi-algebraic triangulation of $\Omega_{\mathcal{Q}}$, which is compatible with the subsets $\Omega_{\mathcal{Q}^{\prime}}$ for every $\mathcal{Q}^{\prime} \subset \mathcal{Q}$, and such that for any simplex $\sigma$ of $\Delta_{\mathcal{Q}}$, index $(\omega P)$, as well as the multiplicities of the eigenvalues of $\omega P$, stay invariant as $\omega$ varies over $h_{\mathcal{Q}}(\sigma)$. Note that by a simplex of certain dimension we mean an open simplex of that dimension. The following proposition relates the homotopy type of $\varphi_{1, \mathcal{Q}}^{-1}\left(h_{\mathcal{Q}}(\sigma)\right)$ to that of a single fiber.

Proposition 4. For any simplex $\sigma \in \Delta_{\mathcal{Q}}$ and $\omega \in h_{\mathcal{Q}}(\sigma), \varphi_{1, \mathcal{Q}}^{-1}\left(h_{\mathcal{Q}}(\sigma)\right)$ is homotopy equivalent to $\varphi_{1, \mathcal{Q}}^{-1}(\omega)$, and both these spaces have the homotopy type of the sphere $\mathbf{S}^{k-\operatorname{index}(\omega P)}$.

Proof. Let $i=\operatorname{index}(\omega P)$. Since index $(\omega P)$ is invariant as $\omega$ varies over $h_{\mathcal{Q}}(\sigma)$, the quadratic forms $\omega P$ has exactly $i$ negative eigen-values for each $\omega \in h_{\mathcal{Q}}(\sigma)$. Let $M(\sigma, \omega) \subset \mathrm{R}^{k+1}$ be the orthogonal complement to the linear span of the corresponding eigen-vectors, and let $B(\sigma, \omega)=M(\sigma, \omega) \cap \mathbf{S}^{k}$. Clearly, $M(\sigma, \omega)$ and $B(\sigma, \omega)$ vary continuously with $\omega$, and $\varphi_{1, \mathcal{Q}}^{-1}(\omega)$ can be retracted to the set $\{\omega\} \times B(\sigma, \omega)$. Finally, since $h_{\mathcal{Q}}(\sigma)$ is contractible to $\omega$, its clear that $\varphi_{1, \mathcal{Q}}^{-1}\left(h_{\mathcal{Q}}(\sigma)\right)$ retracts to $\{\omega\} \times B(\sigma, \omega)$ and the latter has the homotopy type of $\mathbf{S}^{k-\text { index }(\omega P)}$ by Lemma 5.1.

Our next goal is to construct a cell complex homotopy equivalent to $B_{\mathcal{Q}}$ obtained by glueing together certain regular cell complexes, $\mathcal{K}(\sigma)$, where $\sigma \in \Delta_{\mathcal{Q}}$.

Let $1 \geq \varepsilon_{0} \gg \varepsilon_{1} \gg \cdots \gg \varepsilon_{s} \gg 0$ be infinitesimals. For $\eta \in \Delta_{\mathcal{Q}}$, we denote by $C_{\eta}$ the subset of $\bar{\eta}$ defined by,

$$
C_{\eta}=\left\{x \in \bar{\eta} \mid \text { and } \operatorname{dist}(x, \theta) \geq \varepsilon_{\operatorname{dim}(\theta)} \text { for all } \theta \prec \sigma\right\} .
$$

Now, let $\sigma \prec \eta$ be two simplices of $\Delta_{\mathcal{Q}}$. We denote by $C_{\sigma, \eta}$ the subset of $\bar{\eta}$ defined by,

$$
C_{\sigma, \eta}=\left\{x \in \bar{\eta} \mid \operatorname{dist}(x, \sigma) \leq \varepsilon_{\operatorname{dim}(\sigma)} \text {, and } \operatorname{dist}(x, \theta) \geq \varepsilon_{\operatorname{dim}(\theta)} \text { for all } \theta \prec \sigma\right\} \text {. }
$$




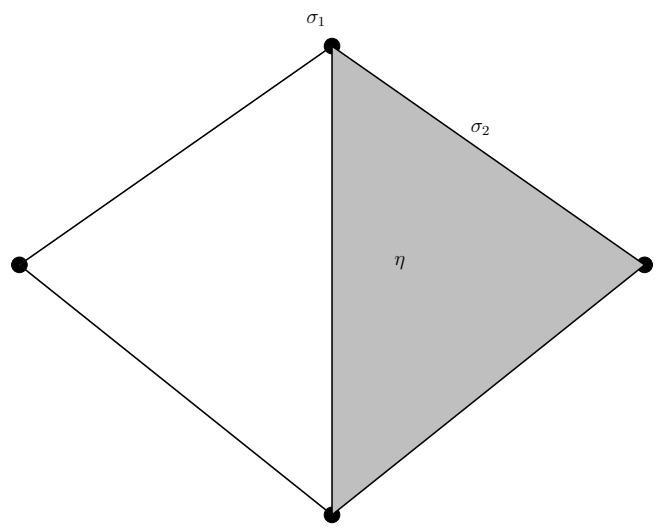

Fig. 1. The complex $\Delta_{\mathcal{Q}}$.

Note that,

$$
\left|\Delta_{\mathcal{Q}}\right|=\bigcup_{\sigma \in \Delta_{\mathcal{Q}}} C_{\sigma} \cup \bigcup_{\sigma, \eta \in \Delta_{\mathcal{Q}}, \sigma \prec \eta} C_{\sigma, \eta} .
$$

Also, observe that the various $C_{\eta}$ 's and $C_{\sigma, \eta}$ 's are each homeomorphic to a ball, and moreover all non-empty intersections between them also have the same property. Thus, the union of the $C_{\eta}$ 's and $C_{\sigma, \eta}$ 's together with the non-empty intersections between them form a regular cell complex, $\mathcal{C}\left(\Delta_{\mathcal{Q}}\right)$, whose underlying topological space is $\left|\Delta_{\mathcal{Q}}\right|$ (see Figures 1 and 2).

We now associate to each $C_{\sigma}$ (respectively, $C_{\sigma, \eta}$ ) a regular cell complex, $\mathcal{K}(\sigma)$, (respectively, $\mathcal{K}(\sigma, \eta))$ homotopy equivalent to $\varphi_{1, \mathcal{Q}}^{-1}\left(h_{\mathcal{Q}}\left(C_{\sigma}\right)\right)$ (respectively, $\varphi_{1, \mathcal{Q}}^{-1}\left(h_{\mathcal{Q}}\left(C_{\sigma, \eta}\right)\right)$.

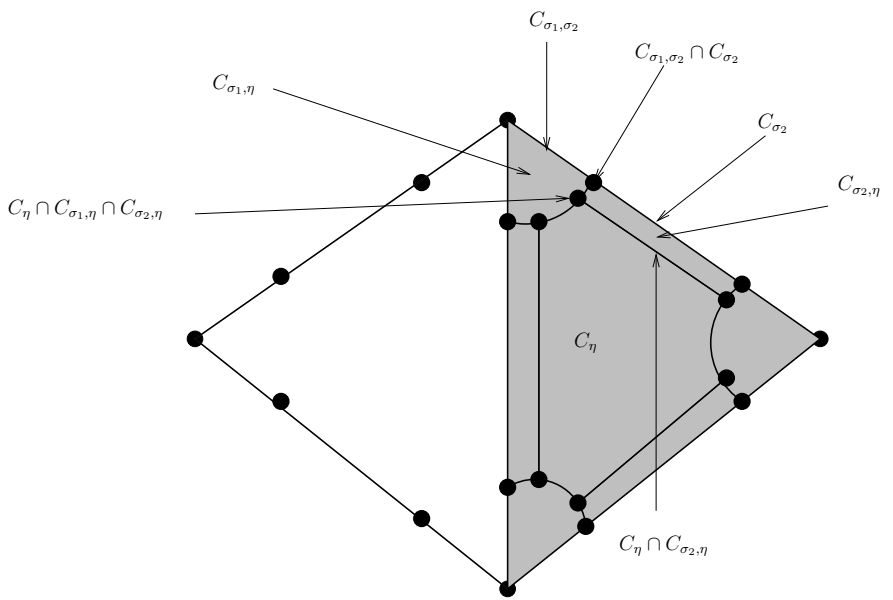

Fig. 2. The corresponding complex $\mathcal{C}\left(\Delta_{\mathcal{Q}}\right)$. 
For each $\sigma \in \Delta_{\mathcal{Q}}$, and $\omega \in h_{\mathcal{Q}}(\sigma)$, let

$$
\begin{gathered}
\lambda_{0}^{\sigma}(\omega)=\cdots=\lambda_{i_{0}}^{\sigma}(\omega)<\lambda_{i_{0}+1}^{\sigma}(\omega)=\cdots=\lambda_{i_{1}}^{\sigma}(\omega)<\cdots<\lambda_{i_{p-1}+1}^{\sigma}(\omega)=\cdots= \\
\lambda_{i_{p}}^{\sigma}(\omega)<0=\cdots=\lambda_{i_{p+1}}^{\sigma}(\omega)<\cdots=\cdots=\lambda_{k}^{\sigma}(\omega)
\end{gathered}
$$

denote the eigenvalues of $\omega P$. Here, index $(\omega P)=i_{p}+1$. Also, since the multiplicities of the eigenvalues do not change as $\omega$ varies over $h_{\mathcal{Q}}(\sigma)$, the block structure, $\left[0, \ldots, i_{0}\right],\left[i_{0}+1, \ldots, i_{1}\right], \ldots,[\cdot, \ldots, k]$ also does not change as $\omega$ varies over $h_{\mathcal{Q}}(\sigma)$. For $0 \leq j \leq p$, let $M^{j}(\sigma, \omega)$ denote the subspace of $\mathrm{R}^{k+1}$ orthogonal to the subspace spanned by the eigenvectors corresponding to the eigenvalues $\lambda_{0}^{\sigma}(\omega)=\cdots=\lambda_{i_{0}}^{\sigma}(\omega)<\lambda_{i_{0}+1}^{\sigma}(\omega)=\cdots=\lambda_{i_{1}}^{\sigma}(\omega)<\cdots<\lambda_{i_{p-1}+1}^{\sigma}(\omega)=\cdots=$ $\lambda_{i_{j}}^{\sigma}(\omega)$, and let $M(\sigma, \omega)=M^{p}(\sigma, \omega)$. Since the eigenvalues vary continuously and their multiplicities do not change as $\omega$ varies over $h_{\mathcal{Q}}(\sigma)$, the flag of subspaces $M^{0}(\sigma, \omega) \supset \cdots \supset M^{p}(\sigma, \omega)$ also varies continuously over $h_{\mathcal{Q}}(\sigma)$.

For each $\sigma \in \Delta_{\mathcal{Q}}$, and $\omega \in h_{\mathcal{Q}}(\sigma)$, let $\left\{e_{0}(\sigma, \omega), \ldots, e_{k}(\sigma, \omega)\right\}$, be a continuously varying orthonormal basis of $\mathrm{R}^{k+1}$, computed using a parametrized version of Gram-Schmidt orthogonalization algorithm, such that $e_{i}(\sigma, \omega), \ldots, e_{k}(\sigma, \omega)$, form an orthonormal basis of $M^{p}(\sigma, \omega)=M(\sigma, \omega)$, where $i=\operatorname{index}(\omega P)$. Since the number and the degrees of the polynomials defining the triangulation $\Delta_{\mathcal{Q}}$ are bounded by $k^{2^{O(\ell)}}$, and the complexity of Gram-Schmidt orthogonalization is polynomial in the size of the input matrix, it is clear that the univariate representations defining the parametrized orthonormal basis $\left\{e_{0}(\sigma, \omega), \ldots, e_{k}(\sigma, \omega)\right\}$, have complexity bounded by $k^{2^{O(\ell)}}$. After having computed the orthonormal basis, $\left\{e_{0}(\sigma, \omega), \ldots, e_{k}(\sigma, \omega)\right\}$, we extend it continuously to each $C_{\sigma, \eta}$ for $\eta$ with $\sigma \prec \eta$, satisfying the condition that

$$
M(\sigma, \omega) \subset \operatorname{span}\left(e_{i}(\sigma, \omega), \ldots, e_{k}(\sigma, \omega)\right) .
$$

This extension can be done in a consistent manner because the first $i$ eigenvalues, $\lambda_{0}(\omega P), \ldots, \lambda_{i-1}(\omega P)$ of $\omega P$ stay negative, and $\lambda_{i-1}(\omega P)<\lambda_{i}(\omega P)$ for $\omega$ in any infinitesimal neighborhood of $h_{\mathcal{Q}}\left(C_{\sigma}\right)$. Thus, the linear subspace of $\mathrm{R}^{k}$ orthogonal to the eigenspaces corresponding to the eigenvalues, $\lambda_{0}(\omega P), \ldots, \lambda_{i-1}(\omega P)$ is well-defined and varies continuously with $\omega$ in any infinitesimal neighborhood of $h_{\mathcal{Q}}\left(C_{\sigma}\right)$.

The orthonormal basis

$$
\left\{e_{0}(\sigma, \omega), \ldots, e_{k}(\sigma, \omega)\right\},
$$

determines a complete flag of subspaces, $\mathcal{F}(\sigma, \omega)$, consisting of

$$
\begin{gathered}
L^{0}(\sigma, \omega)=0, \\
L^{1}(\sigma, \omega)=\operatorname{span}\left(e_{k}(\sigma, \omega)\right), \\
L^{2}(\sigma, \omega)=\operatorname{span}\left(e_{k}(\sigma, \omega), e_{k-1}(\sigma, \omega)\right), \\
\vdots \\
L^{k+1}(\sigma, \omega)=\mathrm{R}^{k+1} .
\end{gathered}
$$


For $0 \leq j \leq k$, let $c_{j}^{+}(\sigma, \omega)$ (respectively, $\left.c_{j}^{-}(\sigma, \omega)\right)$ denote the $(k-j)$ dimensional cell consisting of the intersection of the $L^{k-j+1}(\sigma, \omega)$ with the unit hemisphere in $\mathbf{R}^{k+1}$ defined by $\left\{x \in \mathbf{S}^{k} \mid\left\langle x, e_{j}(\sigma, \omega)\right\rangle \geq 0\right\}$ (respectively, $\{x \in$ $\left.\left.\mathbf{S}^{k} \mid\left\langle x, e_{j}(\sigma, \omega)\right\rangle \leq 0\right\}\right)$.

The regular cell complex $\mathcal{K}(\sigma)$ (as well as $\mathcal{K}(\sigma, \eta)$ ) is defined as follows.

The cells of $\mathcal{K}(\sigma)$ are $\left\{(x, \omega) \mid x \in c_{j}^{ \pm}(\sigma, \omega), \omega \in h_{\mathcal{Q}}(c)\right\}$, where index $(\omega P) \leq$ $j \leq k$, and $c \in \mathcal{C}\left(\Delta_{\mathcal{Q}}\right)$ is either $C_{\sigma}$ itself, or a cell contained in the boundary of $C_{\sigma}$.

Similarly, the cells of $\mathcal{K}(\sigma, \eta)$ are $\left\{(x, \omega) \mid x \in c_{j}^{ \pm}(\sigma, \omega), \omega \in h_{\mathcal{Q}}(c)\right\}$, where $\operatorname{index}(\omega P) \leq j \leq k, c \in \mathcal{C}\left(\Delta_{\mathcal{Q}}\right)$ is either $C_{\sigma, \eta}$ itself, or a cell contained in the boundary of $C_{\sigma, \eta}$.

Our next step is to obtain cellular subdivisions of each non-empty intersection amongst the spaces associated to the complexes constructed above, and thus obtain a regular cell complex, $\mathcal{K}\left(B_{\mathcal{Q}}\right)$, homotopy equivalent to $B_{\mathcal{Q}}$.

First notice that $\left|\mathcal{K}\left(\sigma^{\prime}, \eta^{\prime}\right)\right|$ (respectively, $\left.|\mathcal{K}(\sigma)|\right)$ has a non-empty intersection with $|\mathcal{K}(\sigma, \eta)|$ only if $C_{\sigma^{\prime}, \eta^{\prime}}$ (respectively, $\left.C_{\sigma^{\prime}}\right)$ intersects $C_{\sigma, \eta}$.

Let $C$ be some non-empty intersection amongst the $C_{\sigma}$ 's and $C_{\sigma, \eta}$ 's, that is $C$ is a cell of $\mathcal{C}\left(\Delta_{\mathcal{Q}}\right)$. Then, $C \subset \eta$ for a unique simplex $\eta \in \Delta_{\mathcal{Q}}$, and

$$
C=C_{\sigma_{1}, \eta} \cap \cdots \cap C_{\sigma_{p}, \eta},
$$

with $\sigma_{1} \prec \sigma_{2} \prec \cdots \prec \sigma_{p} \prec \eta$ and $p \leq \# \mathcal{Q}+1$.

Consider $\omega \in h_{\mathcal{Q}}(C)$. We have $p$ different flags,

$$
\mathcal{F}\left(\sigma_{1}, \omega\right), \ldots, \mathcal{F}\left(\sigma_{p}, \omega\right),
$$

giving rise to $p$ independent regular cell decompositions of $B(\omega, \eta)=$ $M(\omega, \eta) \cap \mathbf{S}^{k}$.

There is a unique smallest regular cell complex, $\mathcal{K}^{\prime}(C, \omega)$, that refines all these cell decompositions. The cells of this cell decomposition consists of the following. Let $L \subset M(\omega, \eta)$ be any linear subspace of dimension $m, 0 \leq m \leq k+1$, which is an intersection, of linear subspaces $L_{1}, \ldots, L_{p}$, where $L_{i} \in \mathcal{F}\left(\sigma_{i}, \omega\right), 1 \leq i \leq p$. The elements of the flags, $\mathcal{F}\left(\sigma_{1}, \omega\right), \ldots, \mathcal{F}\left(\sigma_{p}, \omega\right)$ of dimensions $m+1$, partition $L$ into polyhedral cones of various dimensions. The union of the sets of intersections of these cones with $\mathbf{S}^{k}$, over all such subspaces $L \subset M(\omega, \eta)$, are the cells of $\mathcal{K}^{\prime}(C, \omega)$. Figure 3 illustrates the refinement described above in case of two flags in $\mathrm{R}^{3}$.

We now triangulate $h_{\mathcal{Q}}(C)$, using the algorithm implicit in Theorem 3.2 (Triangulation), such that the combinatorial type of the arrangement of flags,

$$
\mathcal{F}\left(\sigma_{1}, \omega\right), \ldots, \mathcal{F}\left(\sigma_{p}, \omega\right)
$$

and hence the cell decomposition $\mathcal{K}^{\prime}(C, \omega)$, stays invariant over the image, $h_{C}(\theta)$, of each simplex, $\theta$, of this triangulation. More precisely, we first compute a family of polynomials, $\mathcal{A}_{C} \subset \mathrm{R}\left[Z_{1}, \ldots, Z_{\ell}\right]$ whose signs at $\omega$ determine the combinatorial type of the corresponding arrangement of flags. It is easy to verify that the number and degrees of the polynomials in the family $\mathcal{A}_{C}$ is bounded by $k^{2^{o(\ell)}}$. We 


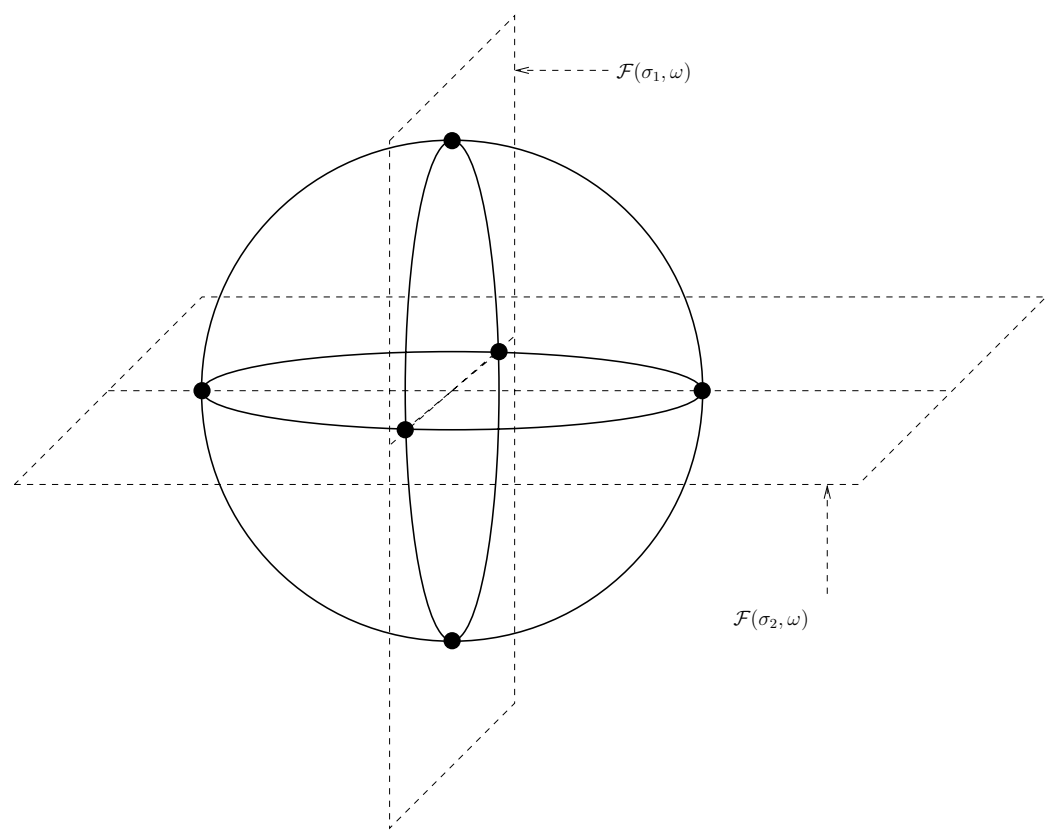

Fig. 3. The cell complex $\mathcal{K}^{\prime}(C, \omega)$.

then use the algorithm implicit in Theorem 3.2 (Triangulation), with $\mathcal{A}_{C}$ as input, to obtain the required triangulation.

The closures of the sets

$$
\left\{(\omega, x) \mid x \in c \in \mathcal{K}^{\prime}(C, \omega), \omega \in h_{\mathcal{Q}}\left(h_{C}(\theta)\right)\right\}
$$

constitute a regular cell complex, $\mathcal{K}(C)$, which is compatible with the regular cell complexes $\mathcal{K}\left(\sigma_{1}\right), \ldots, \mathcal{K}\left(\sigma_{p}\right)$.

The following proposition gives an upper bound on the size of the complex $\mathcal{K}(C)$. We use the notation introduced in the previous paragraph.

Proposition 5. For each $\omega \in h_{\mathcal{Q}}(C)$, the number of cells in $\mathcal{K}^{\prime}(C, \omega)$ is bounded by $k^{O(\ell)}$. Moreover, the number of cells in the complex $\mathcal{K}(C)$ is bounded by $k^{2^{o(\ell)}}$.

Proof. The first part of the proposition follows from the fact that there are at most $k^{\# \mathcal{Q}+1}=k^{\ell+1}$ choices for the linear space $L$ and the number of $(m-1)$ dimensional cells contained in $L$ is bounded by $2^{\ell}$ (which is an upper bound on the number of full dimensional cells in an arrangement of at most $\ell$ hyperplanes). The second part is a consequence of the complexity estimate in Theorem 3.2 (Triangulation) and the bounds on number and degrees of polynomials in the family $\mathcal{A}_{C}$ stated above. 
We denote by $\mathcal{K}\left(B_{\mathcal{Q}}\right)$, the union of all the complexes $\mathcal{K}(C)$ constructed above, noting that by construction, $\mathcal{K}\left(B_{\mathcal{Q}}\right)$ is a regular cell complex.

Proposition 6. $\left|\mathcal{K}\left(B_{\mathcal{Q}}\right)\right|$ is homotopy equivalent to $B_{\mathcal{Q}}$.

Proof. We first show that $B_{\mathcal{Q}}$ is homotopy equivalent to a subset $B_{\mathcal{Q}}^{\prime} \subset B_{\mathcal{Q}}$ as follows. For each simplex $\sigma \in \Delta_{\mathcal{Q}}$ of the largest dimension $\ell$, we use the retraction used in the proof of Proposition 4 , to retract $\varphi_{1}^{-1}\left(h_{\mathcal{Q}}\left(\operatorname{relint}\left(C_{\sigma}\right)\right)\right)$ to the set $\left\{(\omega, x) \quad \mid \omega \in \operatorname{relint}\left(C_{\sigma}\right), x \in B(\omega, \sigma)\right\}$. In this way we obtain a semialgebraic set, $X_{\ell}^{\prime}$, which is a deformation retract of $\operatorname{Ext}\left(B_{\mathcal{Q}}, \mathrm{R}\left\langle\varepsilon_{0}, \ldots, \varepsilon_{\ell-1}\right\rangle\right)$. Let $X_{\ell}=\lim _{\varepsilon_{\ell-1}} X_{\ell}^{\prime}$. Notice that in the definition of $X_{\ell}^{\prime}$, if we replace $\varepsilon_{\ell-1}$ by a variable $t$ and denote the corresponding set by $X_{\ell, t}^{\prime}$, then for all $0<t<t^{\prime}, X_{\ell, t}^{\prime} \subset X_{\ell, t^{\prime}}^{\prime}$ and each $X_{\ell, t}$ is closed and bounded. It then follows (see Lemma 16.17 in [9]) that $\operatorname{Ext}\left(X_{\ell}, \mathrm{R}\left\langle\varepsilon_{0}, \ldots, \varepsilon_{\ell-1}\right\rangle\right)$ has the same homotopy type as $X_{\ell}^{\prime}$, and hence $X_{\ell}$ has the same homotopy type as $\operatorname{Ext}\left(B_{\mathcal{Q}}, \mathrm{R}\left\langle\varepsilon_{0}, \ldots, \varepsilon_{\ell-2}\right\rangle\right)$.

Now repeat the process using the $(\ell-1)$-dimensional simplices and so on, to finally obtain $X_{0}=B_{\mathcal{Q}}^{\prime}$, which by construction has the same homotopy type as $B_{\mathcal{Q}}$. Finally, (again using Lemma 16.17 in [9]) we also have that $X_{0}=\lim _{\varepsilon_{0}}\left|\mathcal{K}\left(B_{\mathcal{Q}}\right)\right|$ and $\operatorname{Ext}\left(X_{0}, \mathrm{R}\left\langle\varepsilon_{0}, \ldots, \varepsilon_{\ell-1}\right\rangle\right)$ has the same homotopy type as $\left|\mathcal{K}\left(B_{\mathcal{Q}}\right)\right|$.

We also have,

Proposition 7. The number of cells in the cell complex $\mathcal{K}\left(B_{\mathcal{Q}}\right)$ is bounded by $k^{2^{O(\ell)}}$.

Proof. The proposition is a consequence of Proposition 5 and the fact that the number of cells in the complex $\mathcal{C}\left(\Delta_{\mathcal{Q}}\right)$ is bounded by $k^{{ }^{o(\ell)}}$.

We now define,

$$
\mathcal{M}_{\mathcal{Q}}^{\bullet}=C^{\bullet}\left(\mathcal{K}\left(B_{\mathcal{Q}}\right),\right.
$$

where $C^{\bullet}\left(\mathcal{K}\left(B_{\mathcal{Q}}\right)\right.$ is the cellular co-chain complex of the regular cell complex $\mathcal{K}\left(B_{\mathcal{Q}}\right)$.

Let $\mathcal{H}\left(T_{\mathcal{Q}}\right)$ (resp. $\mathcal{H}\left(B_{\mathcal{Q}}\right)$ ) be a suitably fine cellular subdivision of $T_{\mathcal{Q}}$ (resp. $\left.B_{\mathcal{Q}}\right)$ and let

$$
\varphi_{2, \mathcal{Q}}^{\prime}: C_{\bullet}\left(\mathcal{H}\left(B_{\mathcal{Q}}\right)\right) \rightarrow C_{\bullet}\left(\mathcal{H}\left(T_{\mathcal{Q}}\right)\right)
$$

be the homomorphism induced by a cellular map, which is a cellular approximation of $\varphi_{2, \mathcal{Q}}$.

Let $\varphi_{\mathcal{Q}}:\left|\mathcal{K}\left(B_{\mathcal{Q}}\right)\right| \rightarrow B_{\mathcal{Q}}$ denote the homotopy equivalence shown to exist by Proposition 6 above and let

$$
\varphi_{\mathcal{Q}}^{\prime}: C_{\bullet}\left(\mathcal{K}^{\prime}\left(B_{\mathcal{Q}}\right)\right) \rightarrow C_{\bullet}\left(\mathcal{H}\left(B_{\mathcal{Q}}\right)\right)
$$


be the homomorphism induced by a cellular approximation to $\varphi_{\mathcal{Q}}$, where $\mathcal{K}^{\prime}\left(B_{\mathcal{Q}}\right)$ is a cellular subdivision of the complex $\mathcal{K}\left(B_{\mathcal{Q}}\right)$.

Since, each cell of $\mathcal{K}\left(B_{\mathcal{Q}}\right)$ is a union of cells of $\mathcal{K}^{\prime}\left(B_{\mathcal{Q}}\right)$, there is a natural homomorphism

$$
\theta_{\mathcal{Q}}: C_{\bullet}\left(\mathcal{K}\left(B_{\mathcal{Q}}\right)\right) \rightarrow C_{\bullet}\left(\mathcal{K}^{\prime}\left(B_{\mathcal{Q}}\right)\right)
$$

obtained by sending each $p$-dimensional cell of $\mathcal{K}\left(B_{\mathcal{Q}}\right)$ to the sum of $p$-dimensional cells of $\mathcal{K}^{\prime}\left(B_{\mathcal{Q}}\right)$ contained in it, for every $p \geq 0$. It is a standard fact that $\theta_{\mathcal{Q}}$ and its dual, $\check{\theta}_{\mathcal{Q}}$, are quasi-isomorphisms.

Let

$$
\psi_{\mathcal{Q}}=\check{\theta}_{\mathcal{Q}} \circ \check{\varphi}_{\mathcal{Q}}^{\prime} \circ \check{\varphi}_{2, \mathcal{Q}}^{\prime}: C^{\bullet}\left(\mathcal{H}\left(T_{\mathcal{Q}}\right)\right) \rightarrow C^{\bullet}\left(\mathcal{K}\left(B_{\mathcal{Q}}\right)\right),
$$

where $\check{\varphi}_{\mathcal{Q}}^{\prime}$ (resp. $\check{\varphi}_{2, \mathcal{Q}}^{\prime}$ ) is the dual homomorphism of $\varphi_{\mathcal{Q}}^{\prime}$ (resp. $\varphi_{2, \mathcal{Q}}^{\prime}$ ).

Proposition 8. For $0 \leq i \leq k-1$, the induced homomorphisms,

$$
\psi_{\mathcal{Q}}^{*}: H^{i}\left(C^{\bullet}\left(\mathcal{H}\left(T_{\mathcal{Q}}\right)\right)\right) \rightarrow H^{i}\left(\mathcal{M}_{\mathcal{Q}}^{\bullet}\right)
$$

are isomorphisms.

Proof. The proof is clear since $\psi_{\mathcal{Q}}$ is a composition of quasi-isomorphisms.

Now let, $\mathcal{B} \subset \mathcal{A} \subset \mathcal{P}$ with $\# \mathcal{A}=\# \mathcal{B}+1<k$.

The simplicial complex $\Delta_{\mathcal{B}}$ is a subcomplex of $\Delta_{\mathcal{A}}$ and hence, $\mathcal{K}\left(B_{\mathcal{B}}\right)$ is a subcomplex of $\mathcal{K}\left(B_{\mathcal{A}}\right)$ and thus there exists a natural homomorphism (induced by restriction),

$$
\varphi_{\mathcal{A}, \mathcal{B}}: \mathcal{M}_{\mathcal{A}}^{\bullet} \rightarrow \mathcal{M}_{\mathcal{B}}^{\bullet}
$$

The complexes $\mathcal{M}_{\mathcal{A}}^{\bullet}, \mathcal{M}_{\mathcal{B}}^{\bullet}$, and the homomorphisms, $\varphi_{\mathcal{A}, \mathcal{B}}, \psi_{\mathcal{A}}, \psi_{\mathcal{B}}$ satisfy

Proposition 9. The diagram

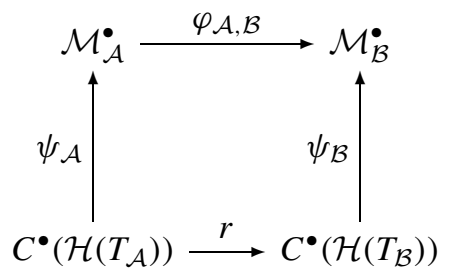

is commutative, where $r$ is the restriction homomorphism.

Proof. Clear from the construction. 
It follows from Proposition 9 that the diagram (6.1) is also commutative.

We denote by

$$
\check{\varphi}_{\mathcal{B}, \mathcal{A}}: \check{\mathcal{M}}_{\mathcal{B}}^{\bullet} \rightarrow \check{\mathcal{M}}_{\mathcal{A}}^{\bullet}
$$

the homomorphism dual to $\varphi_{\mathcal{A}, \mathcal{B}}$. We denote by $\mathcal{D}_{\mathcal{P}}^{\bullet, \bullet}$ the double complex defined by:

$$
\mathcal{D}_{\mathcal{P}}^{p, q}=\bigoplus_{\mathcal{Q} \subset \mathcal{P}, \# \mathcal{Q}=p+1} \check{\mathcal{M}}_{\mathcal{Q}}^{q} .
$$

The vertical differentials,

$$
d: \mathcal{D}_{\mathcal{P}}^{p, q} \rightarrow \mathcal{D}_{\mathcal{P}}^{p, q-1}
$$

are induced componentwise from the differentials of the individual complexes $\check{\mathcal{M}}_{\mathcal{Q}}^{\bullet}$. The horizontal differentials,

$$
\delta: \mathcal{D}_{\mathcal{P}}^{p, q} \rightarrow \mathcal{D}_{\mathcal{P}}^{p+1, q},
$$

are defined as follows: for $a \in \mathcal{D}_{\mathcal{P}}^{p, q}=\bigoplus_{\# \mathcal{Q}=p+1} \check{\mathcal{M}}_{\mathcal{Q}}^{q}$, for each subset $\mathcal{Q}=$ $\left\{P_{i_{0}}, \ldots, P_{i_{p+1}}\right\} \subset \mathcal{P}$ with $i_{0}<\cdots<i_{p+1}$, the $\mathcal{Q}$-th component of $\delta a \in \mathcal{D}_{\mathcal{P}}^{p+1, q}$ is given by,

$$
(\delta a)_{\mathcal{Q}}=\sum_{0 \leq j \leq p+1} \check{\varphi}_{\mathcal{Q}_{j}, \mathcal{Q}}\left(a_{\mathcal{Q}_{j}}\right),
$$

where $\mathcal{Q}_{j}=\mathcal{Q} \backslash\left\{P_{i_{j}}\right\}$.

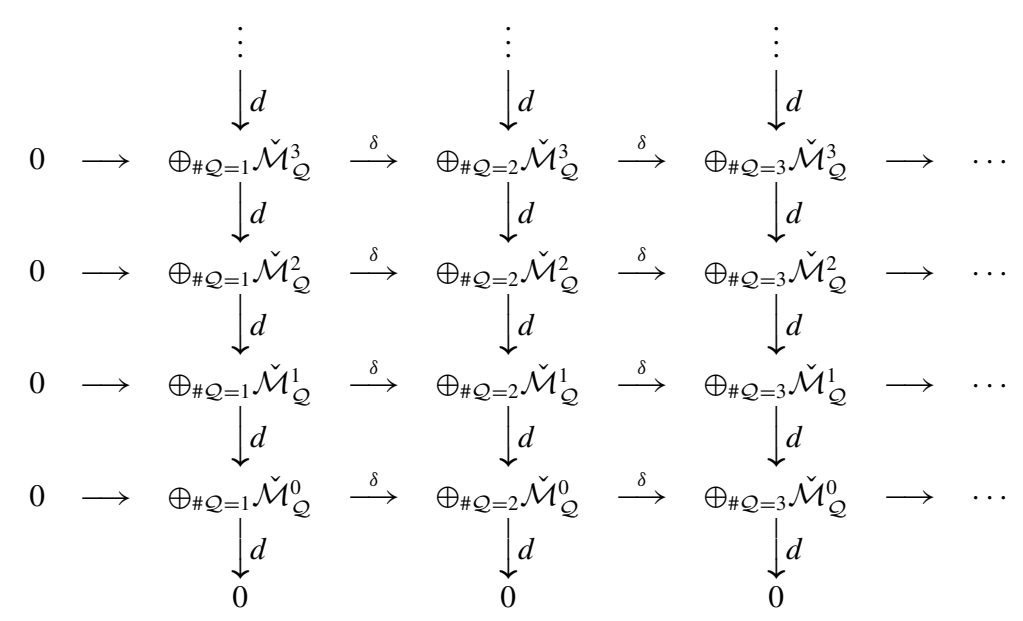

We have the following theorem.

Theorem 6.1. For $0 \leq i \leq k$,

$$
H^{i}(S) \cong H^{i}\left(\operatorname{Tot}^{\bullet}\left(\mathcal{D}_{\mathcal{P}}^{\bullet \bullet \bullet}\right)\right) .
$$


Proof. For each $i, 1 \leq i \leq s$, let $S_{i} \subset \mathbf{S}^{k}$ denote, the set defined on $\mathbf{S}^{k}$ by $P_{i} \leq 0$. Then, $S=\cap_{i=1}^{s} S_{i}$. Choosing a suitably fine triangulation of $\cup_{i=1}^{s} S_{i}$ consider the Mayer-Vietoris double complex, $\mathcal{N}^{\bullet \bullet \bullet}$, as described in Section 3.5. The homomorphisms,

$$
\left.\bigoplus_{\mathcal{Q} \subset \mathcal{P}, \# \mathcal{Q}=p+1} \check{\psi}_{\mathcal{Q}}: \bigoplus_{\mathcal{Q} \subset \mathcal{P}, \# \mathcal{Q}=p+1} \check{\mathcal{M}}_{\mathcal{Q}}^{q} \longrightarrow \underset{\mathcal{Q} \subset \mathcal{P}, \# \mathcal{Q}=p+1}{\bigoplus_{q}} C_{\mathcal{H}}\left(T_{\mathcal{Q}}\right)\right)
$$

give a homomorphism of the double complexes,

$$
\psi: \mathcal{D}_{\mathcal{P}}^{\bullet \bullet \bullet} \longrightarrow \mathcal{N}^{\bullet \bullet \bullet}
$$

By Proposition 8, $\psi$ induces isomporphisms between the $E_{1}$ terms of the two spectral sequences, obtained by taking homology with respect to the vertical differentials. Theorem 3.1 then implies that $\psi$ induces isomorphisms in the associated spectral sequences. But, the second spectral sequence converges to the homology of $S$ by (3.1). The theorem is an immediate consequence.

\section{Algorithms for quadratic forms}

In this section we describe the algorithm for computing the top Betti numbers of a basic semi-algebraic set defined by quadratic forms. We first describe an algorithm for computing the complexes $\check{\mathcal{M}}_{\mathcal{Q}}^{\bullet}$ described in the previous section.

ALGORITHM 1 (Build Complex for Unions).

INPUT:

(A) An integer $\ell, 0 \leq \ell \leq k$.

(B) A quadratic map $P=\left(P_{1}, \ldots, P_{s}\right): \mathrm{R}^{k+1} \rightarrow \mathrm{R}^{s}$ given by $s$ homogeneous quadratic polynomials, $P_{1}, \ldots, P_{s} \in \mathrm{R}\left[X_{0}, \ldots, X_{k}\right]$.

OUTPUT:

(A) For each subset $\mathcal{Q} \subset \mathcal{P}=\left\{P_{1}, \ldots, P_{s}\right\}$, \#Q $\leq \ell+2$ a description of the complex $\check{\mathcal{M}}_{\mathcal{Q}}$, consisting of a basis for each term of the complex and matrices (in this basis) for the differentials.

(B) For each $\mathcal{Q}^{\prime} \subset \mathcal{Q}$, with $\# \mathcal{Q}=\# \mathcal{Q}^{\prime}+1$, matrices for the homomorphisms,

$$
\check{\varphi}_{\mathcal{Q}^{\prime}, \mathcal{Q}}: \check{\mathcal{M}}_{\mathcal{Q}^{\prime}}^{\bullet} \rightarrow \check{\mathcal{M}}_{\mathcal{Q}}^{\bullet} \text {. }
$$

\section{PROCEDURE}

Step 1 For each subset $\mathcal{Q}=\left\{P_{i_{1}}, \ldots, P_{i_{\ell+2}}\right\} \subset \mathcal{P}$, with $\# \mathcal{Q}=\ell+2$, let $P_{\mathcal{Q}}$ be the quadratic map corresponding to the subset $\mathcal{Q}$.

Let $Z_{\mathcal{Q}}=\left(Z_{i_{1}}, \ldots, Z_{i_{\ell+2}}\right)$ and let $M_{\mathcal{Q}}$ be the symmetric matrix corresponding to the quadratic form $Z_{\mathcal{Q}} \cdot P_{\mathcal{Q}}=Z_{i_{1}} P_{i_{1}}+\cdots+Z_{i_{\ell+2}} P_{i_{\ell+2}}$. The entries of $M_{\mathcal{Q}}$ depend linearly on $Z_{i_{1}}, \ldots, Z_{i_{\ell+2}}$. Let,

$$
F\left(Z_{\mathcal{Q}}, T\right)=\operatorname{det}\left(M_{\mathcal{Q}}+T \cdot I_{k+1}\right)=T^{k+1}+C_{k} T^{k}+\cdots+C_{0},
$$


where each $C_{i} \in \mathrm{R}\left[Z_{i_{1}}, \ldots, Z_{i_{\ell+2}}\right]$ is a polynomial of degree at most $k+1$. Let $\mathcal{A}_{\mathcal{Q}}=\left\{C_{0}, \ldots, C_{k}\right\}$.

Step 2 Using the algorithm implicit in Theorem 3.2 (Triangulation), compute a semi-algebraic triangulation,

$$
h_{\mathcal{Q}}: \Delta_{\mathcal{Q}} \rightarrow \Omega_{\mathcal{Q}},
$$

respecting the family $\mathcal{A}_{\mathcal{Q}} \cup \operatorname{Elim}_{T}\left(\left\{F\left(Z_{\mathcal{Q}}, T\right)\right\}\right)$ (see [9] for the definition of Elim), such that for any subset $\mathcal{Q}^{\prime} \subset \mathcal{Q}, \Delta_{\mathcal{Q}^{\prime}}$ is a sub-complex of $\Delta_{\mathcal{Q}}$.

Step 3 Construct the cell complex $\mathcal{C}\left(\Delta_{\mathcal{Q}}\right)$.

Step 4 For each cell $C \in \mathcal{C}\left(\Delta_{\mathcal{Q}}\right)$, compute using a parametrized version of Gram-

Schmidt orthogonalizations, as well as the the algorithm implicit in Theorem

3.2 (Triangulation), the cell complex $\mathcal{K}(C)$ and thus obtain a description of $\mathcal{K}\left(B_{\mathcal{Q}}\right)$

Step 5 Compute the matrices corresponding to the differentials in the complex $\mathcal{M}_{\mathcal{Q}}^{\bullet}=C^{\bullet}\left(\mathcal{K}\left(B_{\mathcal{Q}}\right)\right)$.

Step 6 For $\mathcal{Q}^{\prime} \subset \mathcal{Q} \subset \mathcal{P}$ with $\# \mathcal{Q}=\# \mathcal{Q}^{\prime}+1<k$, compute the matrices for the homomorphisms of complexes,

$$
\check{\varphi}_{\mathcal{Q}^{\prime}, \mathcal{Q}}: \check{\mathcal{M}}_{\mathcal{Q}^{\prime}}^{\bullet} \rightarrow \check{\mathcal{M}}_{\mathcal{Q}}^{\bullet}
$$

in the following way.

The simplicial complex $\mathcal{K}\left(B_{\mathcal{Q}^{\prime}}\right)$ is a subcomplex of $\mathcal{K}\left(B_{\mathcal{Q}}\right)$ by construction. Compute the matrix for the restriction homomorphism,

$$
\varphi_{\mathcal{Q}, \mathcal{Q}^{\prime}}: C^{\bullet}\left(\mathcal{K}\left(B_{\mathcal{Q}}\right)\right) \rightarrow C^{\bullet}\left(\mathcal{K}\left(B_{\mathcal{Q}^{\prime}}\right)\right) .
$$

and output the matrix for the dual homomorphism.

CompleXITy ANALYsis: The complexity of Step 1 is $\sum_{i=0}^{\ell+2}\left(\begin{array}{l}s \\ i\end{array}\right) k^{O(i)}$. The com-

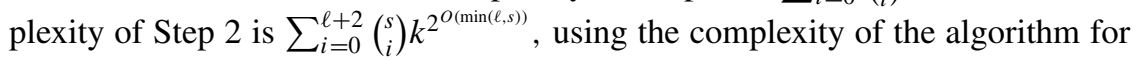
triangulating semi-algebraic sets. It follows from Proposition 7 that the complexities of all the remaining steps are also bounded by $\sum_{i=0}^{\ell+2}\left(\begin{array}{l}s \\ i\end{array}\right) k^{2^{o(\min (\ell, s)}}$.

Proof of Correctness.. It follows from Descarte's rule of signs (see Remark 2.42 , page 41 in [9]) that for any $z \in \Omega_{\mathcal{Q}}$, index $\left(z P_{\mathcal{Q}}\right)$ is equal to the number of sign variations in the sequence $C_{0}(z), \ldots, C_{k}(z),+1$. Thus, the signs of the polynomials $\mathcal{A}_{\mathcal{Q}}=\left\{C_{0}, \ldots, C_{k}\right\}$ determine the index of $z P_{\mathcal{Q}}$. Hence for any simplex $\sigma$ of $\Delta_{\mathcal{Q}}$, index $\left(\omega P_{\mathcal{Q}}\right)$ stays invariant as $\omega$ varies over $h_{\mathcal{Q}}(\sigma)$.

The correctness of the algorithm is now a consequence of Proposition 6 and Proposition 9.

Let $P_{1}, \ldots, P_{s} \in \mathrm{R}\left[X_{0}, \ldots, X_{k}\right]$ be homogeneous quadratic polynomials, and consider the set $S \subset \mathbf{S}^{k}$ defined by, $S=\left\{x \in \mathbf{S}^{k} \mid P_{1}(x) \leq 0, \ldots, P_{s} \leq 0\right\}$.

We will also denote for $1 \leq i \leq s$, by $S_{i}$ the set defined by $\left\{x \in \mathbf{S}^{k} \mid P_{i}(x) \leq 0\right.$. $\}$

Clearly, $S=\cap_{1 \leq i \leq s} S_{i}$. 
ALGORITHM 2 (Computing the highest $\ell$ Betti Numbers: the homogeneous case).

INPUT: A quadratic map $P=\left(P_{1}, \ldots, P_{s}\right): \mathrm{R}^{k+1} \rightarrow \mathrm{R}^{s}$ given by a set, $\mathcal{P}=$

$\left\{P_{1}, \ldots, P_{s}\right\} \subset \mathrm{R}\left[X_{0}, \ldots, X_{k}\right]$, of $s$ homogeneous quadratic polynomials.

OUTPUT: $b_{k}(S), \ldots, b_{k-\ell}(S)$, where $S$ is the set defined by

$$
S=\bigcap_{P \in \mathcal{P}}\left\{x \in \mathbf{S}^{k} \mid P(x) \leq 0\right\} .
$$

PROCEDURE

Step 1 Using Algorithm 1 compute the truncated complex $\mathcal{D}_{\ell}^{\bullet, \bullet}$, i.e.

$$
\begin{array}{rlc}
\mathcal{D}_{\ell}^{p, q} & =\mathcal{D}^{p, q}, & 0 \leq p \leq \ell+1, \quad k-\ell-1 \leq q \leq k, \\
& =0, & \text { otherwise },
\end{array}
$$

Step 2 Compute using linear algebra, the ranks of

$$
H^{i}\left(\operatorname{Tot}^{\bullet}\left(\mathcal{D}_{\ell}^{\bullet, \bullet}\right)\right), \quad k-\ell+1 \leq i \leq k .
$$

Step 3 For each $i, \quad k-\ell \leq i \leq k$, output, $b_{i}(S)=\operatorname{rank}\left(H^{i}\left(\operatorname{Tot}^{\bullet}\left(\mathcal{D}_{\ell}^{\bullet, \bullet}\right)\right)\right)$.

COMPLEXITY ANALYSIS: The number of algebraic operations is clearly bounded by $\sum_{i=0}^{\ell+2}\left(\begin{array}{l}s \\ i\end{array}\right) k^{2^{O(\min (\ell, s))}}$ using the complexity analysis of Algorithm 1.

Proof of Correctness. The correctness of the algorithm is a consequence of the correctness of Algorithm 1 and Theorem 6.1.

Remark 7.1. Suppose that (using Notation from Algorithm 2) $\mathcal{P}^{\prime} \subset \mathcal{P}$ and

$$
S^{\prime}=\bigcap_{P \in \mathcal{P}^{\prime}}\left\{x \in \mathbf{S}^{k} \mid P(x) \leq 0\right\}
$$

and letting $\mathcal{D}_{\ell}^{\prime \bullet \bullet \bullet}$ denote the corresponding complex for $S^{\prime}$, it is clear from the definition that there is a homomorphism, $\Phi_{\mathcal{P}, \mathcal{P}^{\prime}}: \mathcal{D}_{\ell}^{\bullet, \bullet} \rightarrow \mathcal{D}_{\ell}^{\prime \bullet, \bullet}$ defined as follows.

For

$$
\begin{gathered}
\varphi=\bigoplus_{\mathcal{Q} \subset \mathcal{P}, \# \mathcal{Q}=p+1} \varphi_{\mathcal{Q}} \in \mathcal{D}_{\ell}^{p, q}=\bigoplus_{\mathcal{Q} \subset \mathcal{P}, \# \mathcal{Q}=p+1} \check{\mathcal{M}}_{\mathcal{Q}}^{q}, \\
\Phi_{\mathcal{P}, \mathcal{P}^{\prime}}(\varphi)=\bigoplus_{\mathcal{Q} \subset \mathcal{P}^{\prime}, \# \mathcal{Q}=p+1} \varphi_{\mathcal{Q}} .
\end{gathered}
$$

Recall from 6.2 that there exists,

$$
\psi: \mathcal{D}_{\ell}^{\bullet \bullet \bullet} \longrightarrow \mathcal{N}_{\ell}^{\bullet \bullet \bullet}
$$

which induces an isomorphism, $\psi^{*}: H^{*}\left(\operatorname{Tot}^{\bullet}\left(\mathcal{D}_{\ell}^{\bullet, \bullet}\right)\right) \longrightarrow H^{*}\left(\operatorname{Tot}^{\bullet}\left(\mathcal{N}_{\ell}^{\bullet, \bullet}\right)\right)$. 
Denoting by $\mathcal{N}_{\ell}^{\prime \bullet \bullet}$ the (truncated) Mayer-Vietoris complex for $S^{\prime}$ and by $i_{\mathcal{P}, \mathcal{P}^{\prime}}$ : $\mathcal{N}_{\ell}^{\bullet, \bullet} \rightarrow \mathcal{N}_{\ell}^{\prime \bullet \bullet \bullet}$ the inclusion homomorphism, we have the following commutative diagram.

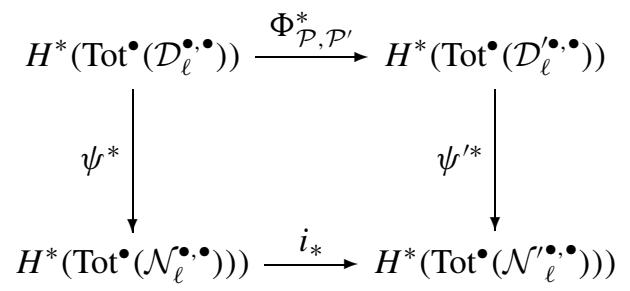

Note that $H^{*}\left(\operatorname{Tot}^{\bullet}\left(\mathcal{N}^{\bullet \bullet \bullet}\right)\right) \cong H_{*}(S)$ and $H^{*}\left(\operatorname{Tot}^{\bullet}\left(\mathcal{N}^{\prime \bullet}, \bullet\right)\right) \cong H_{*}\left(S^{\prime}\right)$.

It is clear that Algorithm 2 can be easily modified to output the complex $\mathcal{D}_{\ell}^{\bullet \bullet}$, by outputting the matrices corresponding to the vertical and horizontal homomorphisms in the chosen bases. Furthermore, given a subset $\mathcal{P}^{\prime} \subset \mathcal{P}$, Algorithm 2 can be made to output both the complexes $\mathcal{D}_{\ell}^{\bullet, \bullet}$ and $\mathcal{D}_{\ell}^{\prime \bullet \bullet \bullet}$ along with the matrices defining the homomorphism $\Phi_{\mathcal{P}, \mathcal{P}^{\prime}}$ with the same complexity bounds. 